\title{
Review
}

\section{Noble metal nanomaterials for the diagnosis and treatment of hematological malignancies}

\author{
Xin Huang, ${ }^{1, *}$, Hasan Md Mahmudul ${ }^{1}$, Zengbei $\mathrm{Li}^{1}$, Xinjie Deng ${ }^{1}$, Xiaozhou $\mathrm{Su}^{1}$, \\ Zhongqing Xiao ${ }^{2}$, Lingyun $\mathrm{Zhao}^{2}$, Ting $\mathrm{Liu}^{2}$, Hongyun $\mathrm{Li}^{2}$ \\ ${ }^{1}$ Department of Light Chemical Engineering, School of Textiles, Zhongyuan University of Technology, 450007 Zhengzhou, Henan, China \\ ${ }^{2}$ Department of Respiratory and Critical Care Medicine, The Fifth Affiliation Hospital of Zhengzhou University, 450052 Zhengzhou, Henan, China \\ *Correspondence: xinhuang@zut.edu.cn (Xin Huang) \\ Academic Editor: Elena Levantini \\ Submitted: 31 August 2021 Revised: 21 October 2021 Accepted: 29 October 2021 Published: 24 January 2022
}

\begin{abstract}
Background: Recently, the incidence of hematological malignancy, such as various leukemias, multiple myeloma and lymphoma, has revealed an increasing tendency, exhibiting a major impact on human health. Most of the available anti-cancer drugs, however, possess high non-targeted accumulation, dosage-associated toxicity, fast elimination, and lack specificity towards tumors, which restrict their utilization in clinical therapy. This extends also to cancer diagnosis where there is a lack of predictive biomarkers. Object: Noble metal nanomaterials (NM NMs) have the potential to overcome these shortcomings due to several characteristics including ease of synthesis, ultra-small size, easy surface modification and specific physicochemical properties. At present, gold-, silver- and platinumbased nanomaterials have been employed in the tracing and treatment of hematopoietic tumors through direct individual endocytosis or in innovative drug delivery systems (DDS) by conjugation with other targeting biomolecules. Purpose: In this mini review, we focus on the use of localized surface plasmon resonance (LSPR)-/surface-enhanced Raman scattering (SERS)- and fluorescence-based diagnosis of NM NMs in the hematological malignancies. Furthermore, the treatment of hematological malignancies utilized the NM NMs or NM NMs-based therapy technology in the chemotherapy, targeted therapy, and photothermal therapy are depicted in depth. The construction of effective and promising NM NMs or NM NMs- dependent theranostic methodology has the potential to provide interdisciplinary knowledge in the development of clinical tracing, diagnosis and treatment of refractory hematological diseases.
\end{abstract}

Keywords: Hematological malignancy; Noble metal nanomaterials; SERS; Bio-imaging; Chemotherapy; Targeted therapy; Photothermal therapy

\section{Introduction}

Cancer as the one of three challenges of modern medicine is reported to be the leading cause of human mortality and the main hindrance to prolonging life expectancy worldwide in the 21 st century [1]. Hematologic malignancies represent malignant tumors of bone marrow, hematopoietic and lymphoid tissues, accounting for $7.2 \%$ of total deaths and $6.5 \%$ of new cancers, based on global cancer statistics in 2018 [2]. Generally, hematological malignancies are divided into three types, namely leukemias, lymphomas and myelomas, and each of these contain multiple subtypes [3-5]. Leukemias include acute lymphoblastic leukemia (ALL), acute myelogenous leukemia (AML), chronic lymphocytic leukemia (CLL), chronic myelogenous leukemia (CML), acute monocytic leukemia (AMoL) and other less common subtypes [6]. Lymphomas contain non-Hodgkin's lymphoma and Hodgkin's lymphoma [7], while myelomas are divided into myelodysplastic syndrome (MDS) and myeloproliferative neoplasms (MPN) [8]. Among these, leukemia is associated with high mortality and morbidity rates in comparison to other subtypes, and three quarters of the cases occur in childhood $[6,9,10]$.
Over the past several years, hemopathology has gained a great development, especially the emergence of numerous new technologies for the diagnosis of hematological malignancies [11]. The World Health Organization (WHO) has reported a complex classification standard for hematological malignancies, which makes the diagnosis more difficult $[3,12]$. The increased use of morphology, immunology, genetics and molecular biology (MIGM) has become important diagnostic methodologies for various hematological malignancies [13]. On the other hand, the treatment of hematological malignancies has also been gradually improved, resulting in significant improvement in complete remission (CR) rates, disease-free survival (DFS) rates and overall survival (OS) rates for patients [14]. Although induction differentiation therapy, autologous stemcell transplantation (SCT), biological immunotherapy, targeted and gene therapy have developed rapidly recently, chemotherapy and radiotherapy are still predominantly used for the treatment of hematological diseases (Fig. 1) $[4,7,15,16]$. However, the traditional combined treatment with chemotherapy and radiotherapy leads to discomfort and irreversible side effects such as hair loss, fatigue, nausea, even infection and organ damage [17-19]. Patients 


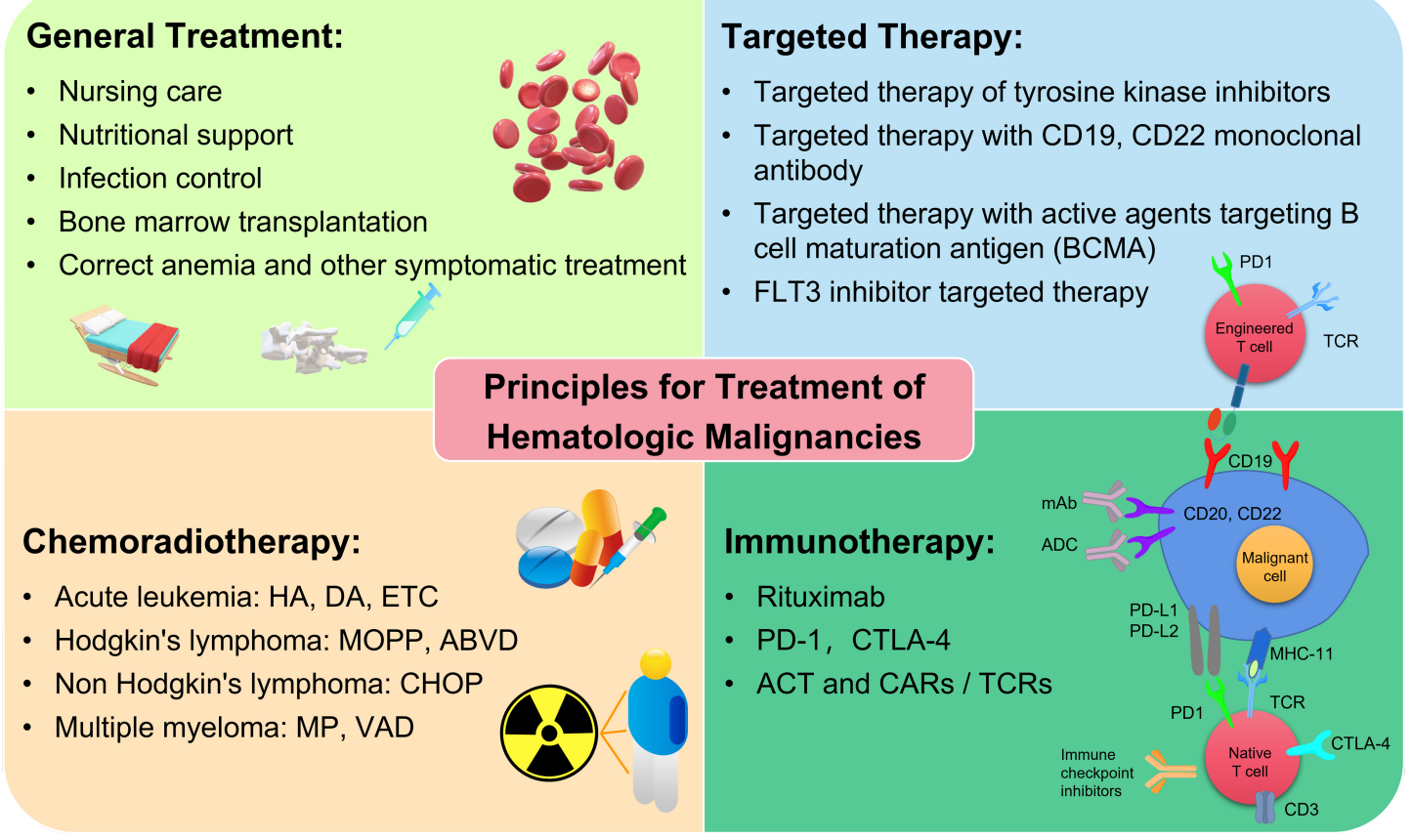

Fig. 1. The schematic principles for treatment of hematological malignancies.

with hematologic malignancy receiving chemotherapeutic drugs have the complication of febrile neutropenia, which dramatically increases the infection rate and mortality [20]. Besides, the pharmacokinetics of chemotherapeutic drugs are suboptimal, leading to inevitable relapse and reduced limitation to their clinical application. Bone marrow transplantation as one form of stem cell transplantation (SCT) represents another choice to cure different hematological malignancies, which has the capacity to achieve better survival rates [21]. Rare match type, few relevant patients and likely complications including graft versus host reaction (GVHD), recurrence, infection, and end organ dysfunction, create prominent resistance to the therapeutic process $[22,23]$. Certainly, some patients are not eligible for SCT or frequently relapse after SCT, therefore, novel treatments still need to be intensively explored.

Monoclonal antibodies alone or combined with chemotherapy, referred to as immunotherapy, have introduced an innovative therapeutic regimen for hematologic malignancies as depicted in Fig. 1 [24-27]. A representative monoclonal antibody is rituximab which has been shown to improve the clinical outcome and efficiently decrease the mortality for the patients with B-cell malignancies [28]. Moreover, some monoclonal antibodies such as bispecific $\mathrm{T}$ cell engagers (BiTEs), cytotoxic Tlymphocyte-associated protein 4 (CTLA-4) inhibitor, and programmed cell death protein 1 (PD-1) inhibitor, regulate the activation of $\mathrm{T}$ cell via the histocompatibilityantigenic peptide complex that expresses a chimeric antigen receptor (CAR), resulting in the eradication of tumor and strengthening of immune response [4,29-32]. Despite the fact that immunotherapy provides considerable hope for patients, these T-cell-engaging therapies are also associated with certain toxicity $[33,34]$. With the development of molecular biologicals, genomic and nano technology, targeted therapy construction of suitable drug delivery systems has become more efficient, safe and represent pharmacokinetic treatment alternatives. Integration of targeted drugs into conventional chemotherapy, radiotherapy and SCT, has become an innovative breakthrough in the traditional treatment of patients with various refractory tumor $[35,36]$. These drugs have a targeted effect on the site of the neoplasm and impede the biological transduction pathways and/or certain oncoproteins to induce the death of carcinoma cells by immune system stimulation or apoptotic effects. Recently, considerable progress has been made on the discovery of targeted therapeutic methods for hematologic malignancies. For example, employing CD20 monoclonal antibodies, fms-related tyrosine kinase 3 (FLT3) inhibitor, and tyrosine kinase inhibitor to target therapy for B-cell lymphoma and CD20 positive leukemia [37], FLT3 positive and high-risk AML [38] and CML [39], respectively. Nevertheless, only few reports showed complete cure or acquired long-term remission due to short biological half-life in circulation and damage to healthy tissues [40]. Chemotherapeutic drugs encapsulated by nano-carriers like liposomes and polymeric micelles, have an ability to retain active substances during transportation towards malignant cells and reduce the exposure of drugs to normal tissues, which has been successfully implemented in some clinical reports [41-43].

In the past decades, metal nanomaterials (M NMs) have received extensive attention in the fields of electronics, catalysis, optics, and biology [44-46]. When the size 
of bulk metal compounds is decreased to the nano range, dramatic physical and chemical properties change due to the quantum size effect, surface effect, and macro-quantum tunnel effect [47]. Metal nanoparticles (M NPs) are generally defined as particle-aggregates with sizes between 2 and 100 nanometers $[48,49]$. As the free electrons are confined related to Fermi wavelength $(<2 \mathrm{~nm})$, M NPs evolve into metal nanoclusters (M NCs) which bridge a "missing link" between M NP and atoms [50-53]. Consequently, M NCs exhibit unique features like discrete electronic state and size-dependent fluorescence because of their discontinuous band structure. Noble metal is one kind of rare and popular material which has an inherent resistance to corrosion and oxidation even under a moist environment [54]. Various noble metal nanomaterials (NM NMs), such as copper nanomaterials ( $\mathrm{Cu} \mathrm{NMs}$ ), palladium nanomaterials (Pd NMs), silver nanomaterials (Ag NMs), platinum nanomaterials (Pt NMs) and gold nanomaterials ( $\mathrm{Au} \mathrm{NMs),}$ have been already studied and exploited in the applications of electrocatalysis, photocatalysis, heterogeneous catalysis, colorimetric sensing and fluorescent imaging. The diagnosis and treatment of tumors rely especially on their extraordinary optical, electronic and surface chemical properties $[46,55,56]$. Moreover, cytotoxicity and biokinetics of NM NMs decide the clinical efficacy of oncotherapy. Typical $\mathrm{Au}, \mathrm{Ag}, \mathrm{Pt}$ NMs demonstrated less toxic for cellular systems and excellent drug kinetics which is related to their chemical nature or surrounding ligands [57-60], ensuring their feasible diagnostic and therapeutic application for malignancies. In this review, we focus on the recent advances in the multifunctional NM NMs for their practical application both in the diagnosis and treatment of hematologic malignancy. The challenges of NM-based theranostic strategy for hematological diseases in future are discussed with a view to providing some new methods and guideline for the hematologist.

\section{The diagnosis of hematological malignancies}

Early and accurate diagnosis of malignancy is one of the most critical points for patients to alleviate mental pain and economic burden [44]. Current cell-based diagnostic tools such as immunocytochemistry, anatomical imaging, pathological examination require sufficient malignant cells, which are usually evident in advanced stages of the disease. X-ray imaging, computerized tomography (CT), positron emission tomography (PET), magnetic resonance imaging (MRI) as the classical anatomical imaging techniques which are associated with intense ionizing radiation or electromagnetic radiation can be harmful to humans [61]. Finding a less harmful alternative tissue imaging methodology in monitoring of cancer is an important issue. Body fluids (blood, urine, saliva) contain potential biomarkers associated with the evolution of cancer cells, such as proteins/peptides, microRNAs (miRNAs), exosomes, circulat- ing tumor DNA (ctDNA), circulating tumor cells (CTCs) represent an approach to such imaging [62-67]. Establishing a rapid and non-invasive approach instead of standard tissue biopsy to test the prognosis agents mentioned above, even in a low concentration, could reduce the risk of metastasis and detect cancer evolution at early stages. In general, NM NMs with unique physicochemical properties and low toxicity are normally employed as sensitive probes or safe contrast agents to achieve the analyte detection and cellular imaging of tumors on account of their specific localized surface plasmon resonance (LSPR), surface-enhanced Raman scattering (SERS), and size-dependent fluorescence.

\subsection{LSPR- or SERS-based diagnosis}

Since the light is incident to the surface of noble metallic NPs, the conduction electrons exhibit specific collective oscillations leading to the emergence of a strong optical absorption and/or scattering peak as shown in Fig. 2A [68]. This unique phenomenon is termed LSPR entirely affected by the NPs' size, shape, dielectric environment and other properties NM NPs, therefore, are frequently used as contrast agents for microscopic imaging or as targeted probes for detection and recognition of tumor cells dependent on the shifts of LSPR spectral peak when the diagnostic objects approach the surface of NPs [69,70]. For example, screening blood samples for leukemia was proposed using nanohole-arrays on plastic (NAP) as a plasmonic sensor and human immunoglobulin kappa and lambda light chains in blood serum as a screening compound [71]. NAP was fabricated by the UV nanoimprinting on thin gold films to form the nanohole arrays. This typical SPR-based nanosensing platform has a capacity to accurately examine the overexpression of light chain antibodies in cancerous blood samples. Cytokines as one kind of immunomodulating protein biomarker, are secreted from immune cells and control cell growth, cell differentiation and immune response [72]. The classical enzyme-linked immunosorbent assay (ELISA) and fluorescent-based detection of cytokines have several disadvantages like time-consuming, complexity, and large sample consumption. A LSPR optofluidic platform device has been integrated with a polydimethylsiloxane (PDMS) supporting layer, a microfluidic layer which traps and incubates cells, and an LSPR sensing layer consisting of $\mathrm{Au}$ NPs connected with tumor necrosis factor (TNF)- $\alpha$ for cytokine detection [73]. This new-type optofluidic system was firstly applied in identifying THP-1 cells (human myeloid leukemia mononuclear cells) utilizing 100 times less sample volume and 3 times less in detection time than ELISA.

If molecules are located on the surface of metallic NPs, the Raman scattering signal is remarkably enhanced, which is defined as SERS (Fig. 2B) [70,74-78]. SERS technology offers a new strategy for both biomolecule detection and intracellular imaging [61]. In addition to high sensitivity, SERS can precisely recognize molecular struc- 
A

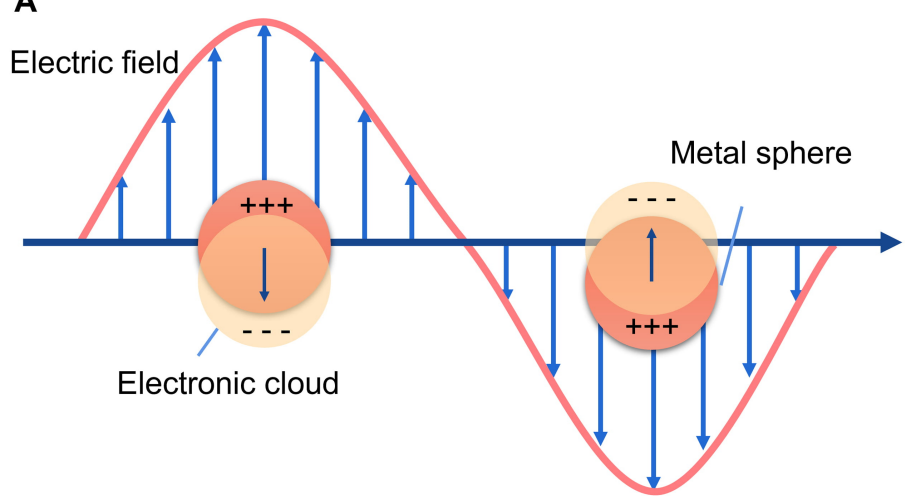

Localized surface plasmon resonance (LSPR)

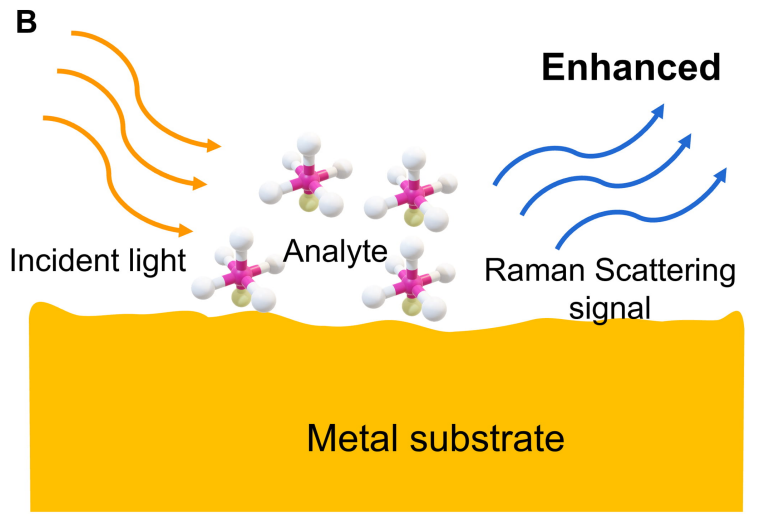

Surface-enhanced Raman scattering (SERS)

Fig. 2. The schematic principle for localized surface plasmon resonance (LSPR), and surface-enhanced Raman scattering (SERS). (A) Since the light is incident to the surface of noble metallic NPs, the conduction electrons exhibit specific collective oscillations leading to the emergence of a strong optical absorption and/or scattering peak. (B) Raman scattering signal is remarkably enhanced when the molecules are located on the surface of metallic naoparticles. Reprinted major modification with permission from [70], Copyright 2011, American Chemical Society.

ture as well as avoid interference from cellular autofluorescence [79]. Lentini et al. [80] constructed Ag NPs assembled phage clone (Ag NPs-EIII1) network as a SERS probe to identify the human histiocytic lymphoma U937 cells in vitro. Once Ag NPs-EIII1 targeted to the U937 cells, a new Raman scattering peak and enhancement intensity appeared, due to the existence of oligosaccharide complexes by means of molecules involved in probe-target interaction. Boca's group initially prepared hollow gold-silver nanoparticles (HNS) and then mixed them with Nile Blue (NB) Raman reporter, modified by PEG to conjugate with antiCD19 monoclonal antibodies [81]. Consequently, SERS active molecules, HNS-NB-PEG-antiCD19, were produced and used for evaluating the uptake and intracellular distribution inside both CD19-positive SKW6.4 cells (EpsteinBarr virus-transformed B lymphocytes, B-cell lymphoma) and CD19-negative OCI-AML3 cell line (AML). The results revealed that HNS-NB-PEG-antiCD19 could selectively targeted and imaged the CD19-positive lymphoma cells, showing the potential for a luciferous assay in the early and precise diagnosis of lymphoblastic cancers.

In summary, LSPR-/SERS-based diagnostic strategies provide sensitive identification of neoplastic indicator and noninvasive imaging of hematologic cells, designed to understand and improve the treatment of hematologic system diseases in advance.

\subsection{Fluorescence-based diagnosis}

Among various types of diagnostic methods, fluorescence-based techniques exhibit a highly sensitive, specific, and time-saving approach for the detection and imaging of various cancers. To achieve the fluorescencebased diagnosis involves combining the effective fluorescent agents such as organic dyes [82], fluorescent proteins [83], semiconductor quantum dots (QDots) [84], carbon nanotubes (CNTs) [85]. Novel NM NCs with discrete electronic state possess molecular-like behaviors and exhibit size-dependent fluorescence from visible to the near infrared (NIR) region [50]. In contrast to classical fluorophores, ultra-fine Au, Ag and Pt nanoclusters (NCs) have stable photobleaching, strong luminescence, and excellent biocompatibility, which has led to great progress in the application of biomolecular detection and cellular labeling for hematological malignancies. Tan's group firstly reported the facile production of multidentate thioether-terminated poly (methacrylic acid) (PTMP-PMAA) stabilized NIR fluorescent $\mathrm{Au}$ NCs with $660 \mathrm{~nm}$ emission wavelength (Fig. 3A) [86]. These Au NCs were able to bio-label both adherent HeLa cells and suspended Jurkat cells. More interesting was the observation that hematopoietic cancer K562 cells had a distinct tendency for internalizing more $\mathrm{Au} \mathrm{NCs}$ than normal cord blood mononuclear cells (CBMC), exhibiting a great potential application in diagnostic detection of hematologic malignancies due to their selective affinity to enter relative mature cells such as granulocytes and lymphocytes (Fig. 3B) [86]. Fluorescent glycine dimers capped Ag NPs with the sizes ranging from 9 to $32 \mathrm{~nm}$ have been produced (Fig. 3C) [87]. This blue fluorescent NPs-ligand system has a high quantum yield of $(5.2 \pm 0.1) \%$ and was subsequently used to bio-image rat basophilic leukemia cells (Fig. 3D) [87]. As another common noble metal, Pt NMs are frequently used as 
catalysts in many chemical reactions based on their high surface activity in nano dimension [88]. However, the fluorescence of ultrafine Pt NCs was developed in the past decades and applied in the metal ion or antibiotic detection, and bio-imaging in vitro [89-93]. Chen et al. [94] developed bifunctional polyethylenimine (PEI)-encased $\mathrm{Pt}$ NCs (short for Pt NCs@PEI, as shown in Fig. 3E), and then co-cultured them with blood cancer K562 and BV173 leukaemia cell lines. This research demonstrated that $\mathrm{Pt}$ NCs smaller than $2 \mathrm{~nm}$ could selectively image K562 and BV173 cells with the internalized proportion at $(97 \pm 4) \%$ and $(95 \pm 4) \%$ respectively, compared to peripheral blood mononucleated cells (PBMCs) at (20 \pm 8$) \%$ (Fig. 3F) [94]. These fluorescent Pt NCs have shown a great potential in the diagnosis of hematopoietic diseases such as leukemia, lymphoma, and myeloma, avoiding the inclusion of extra fluorescent biomarkers.

\section{The treatment of hematological malignancies}

With the rapid development of nanotechnology, NM NMs have the potential to play a crucial role in therapeutic applications for various cancers such as lung carcinoma, prostatic carcinoma, hepatocellular carcinoma and for other tumors. The intrinsic features of NM NMs including ultrafine size, unique optical and electronical properties, especially their easy modifiable surface, offer an opportunity for conjugation with contrast agents, chemotherapeutic agents and physiotherapy agents, to establish innovative theranostic platforms [95]. In this section, we focus on the treatment of hematological malignancies using NM NMs or NM NMs-based curative system in chemotherapy, targeted therapy, and photothermal therapy.

\subsection{Chemotherapy}

As mentioned for chemotherapy, Pt(II)-based chemotherapeutics are traditional and preferred candidates for the clinical treatment of solid tumors [96]. After the approval of the Food and Drug Administration (FDA) in 1978, Pt chemotherapeutic drugs have evolved initially from cisplatin to carboplatin, lobaplatin and oxaliplatin [97,98]. The problems of Pt(II)-based chemotherapeutics including poor pharmacology, systemic toxicities, rapid blood clearance, and side effect like nephrotoxicity, neurotoxicity, ototoxicity, and myelosuppression which strongly restrict their clinic efficiency and usable range, needs to be addressed [99]. Hence, major efforts have been made in exploring the new Pt-based drugs in order to eliminate toxic side effects and drug-resistance of tumors. Recently, the effects of small-sized Pt NPs or Pt NCs as anticancer nanomedicine to augment chemotherapeutic efficacy have been investigated [100,101]. Besides the fluorescent imaging capacity, dual-functional Pt NCs mentioned above showed selective inhibition of hematopoietic K562 and BV173 cancer cells [94]. In contrast to hematopoietic normal cells, these Pt NCs induce a three times higher apoptotic rate in hematopoietic cancer cells. Additionally, immunoblotting was used to confirm the molecular mechanism of Pt NCs'- induced apoptosis and the results suggested that $\mathrm{Pt}$ NCs could induce pro-apoptotic protein expression (p53, PUMA, cleaved caspase) in hematopoietic cancer cells, leading to apoptosis in these cells. Some research also reported the apoptosis mechanism of $\mathrm{Pt}$ NCs-based chemotherapeutics, that is, high surface-active Pt NCs are eroded to an oxidation state caused by interaction with intracellular acidic organelles (endosomes, lysosomes, etc.) whereby Pt ions damage DNA, giving rise to a synergistic effect of both Pt NCs and Pt ions [52].

In addition to conventional $\mathrm{Pt}, \mathrm{Au}$ and $\mathrm{Ag} \mathrm{NMs}$ have also exhibited the induced apoptosis of hematological malignancies and the possibility for them to be developed as novel chemotherapeutics for the effective treatment of hematopoietic system disease, especially AML, lymphoma and multiple myeloma [102-104]. Kumari et al. [105] proposed a green nyctanthes arbortristis mediated synthetic method of Ag NPs with an average size of $22 \mathrm{~nm}$. The different concentrations (5-50 $\mu \mathrm{g} / \mathrm{mL})$ of Ag NPs impacted on cell viability of THP-1 human leukemia cell lines with a half maximal inhibitory concentration (IC50) of $33.5 \mu \mathrm{g} / \mathrm{mL}$. This cytotoxicity in vitro of Ag NPs for leukemia cells was contributed to cell ROS defense mechanisms resulting in cell death. Similarly, the Ag NPs produced in the Brassica rapa extract (NBRE) have the highest antioxidant and cytotoxic activity of 3.53 and $7.38 \mu \mathrm{g} / \mathrm{mL}$ for M-NFS-60 cells (Human Mouse Myelogenous Leukemia carcinoma) and HeLa cells, respectively [106]. Moreover, the solvent effect on Au NPs' size was investigated and dimethyl formamide (DMF) and $N$-methyl-2-pyrrolidone (NMPL) mediated synthesized Au NPs possessed distinct average sizes of 10.2 and $40.4 \mathrm{~nm}$, respectively [107]. The smaller Au NPs had a greater inhibitory effect on K562 cells (88\% cell inhibition) due to their ideal size and easier permeability of cell membranes.

\subsection{Targeted therapy}

On account of the unsatisfactory bioavailability and non-specifical biodistribution of conventional chemotherapeutic drugs, certain molecular ligands like antibodies, proteins (including their fragments), nucleic acids (aptamers), and other receptor ligands (peptides, etc.) impose upon the chemotherapeutics the targeting and selective properties, that is targeted therapy (Fig. 4) [108]. Targeting agents facilitate the NM NMs to specifically reorganize the membrane receptors or antigens on target tumor cells and the type of selective targeting agents greatly determine the physicochemical properties of conjugates. Hence, NM NMs-ligand conjugates (combination with specific targeting agents) are a major way to limit the drug distribution in vivo and target onto the lesion site. Recently, the primary target site of hematologic malignancies is CD19 despite the 

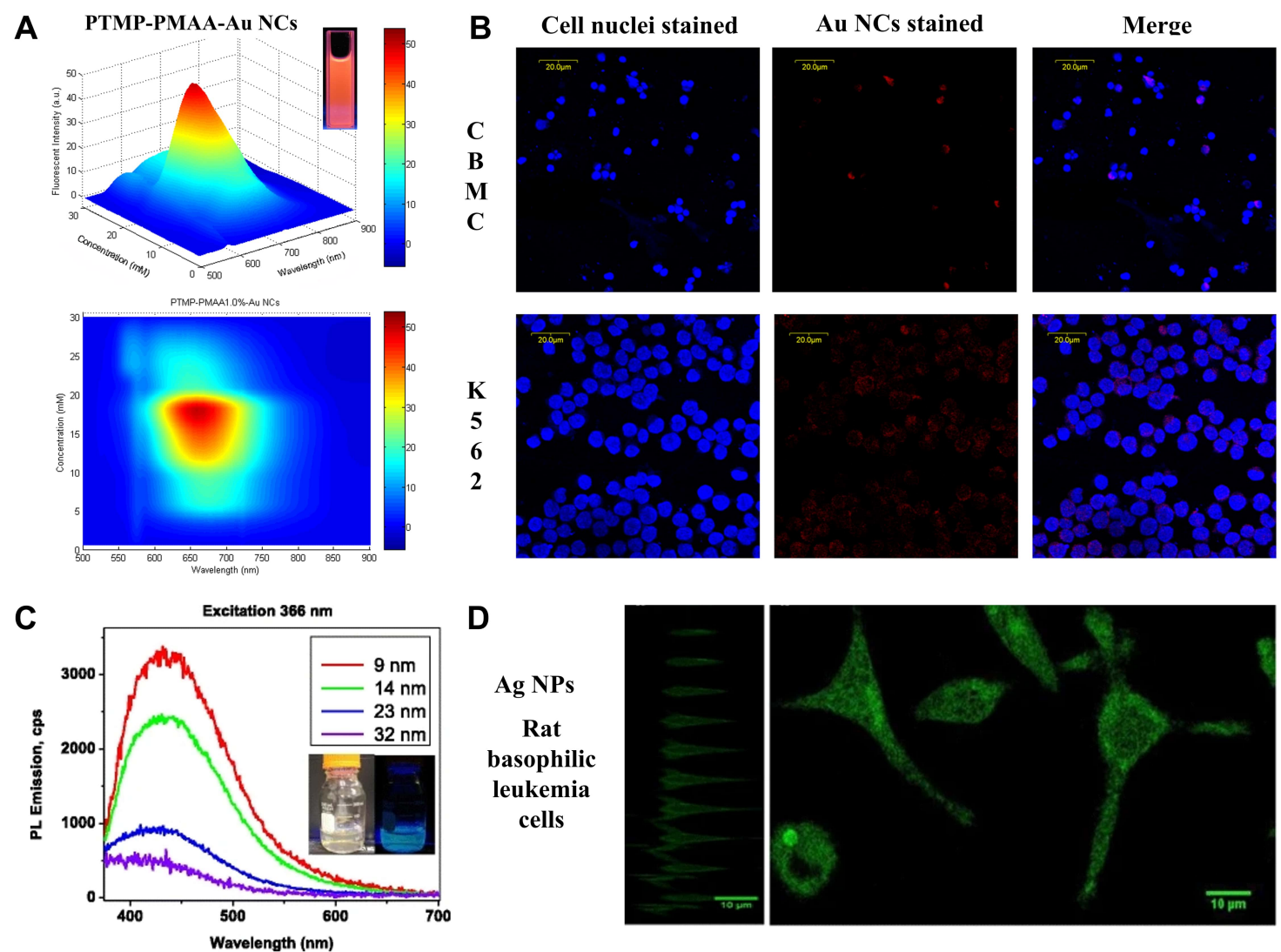

E
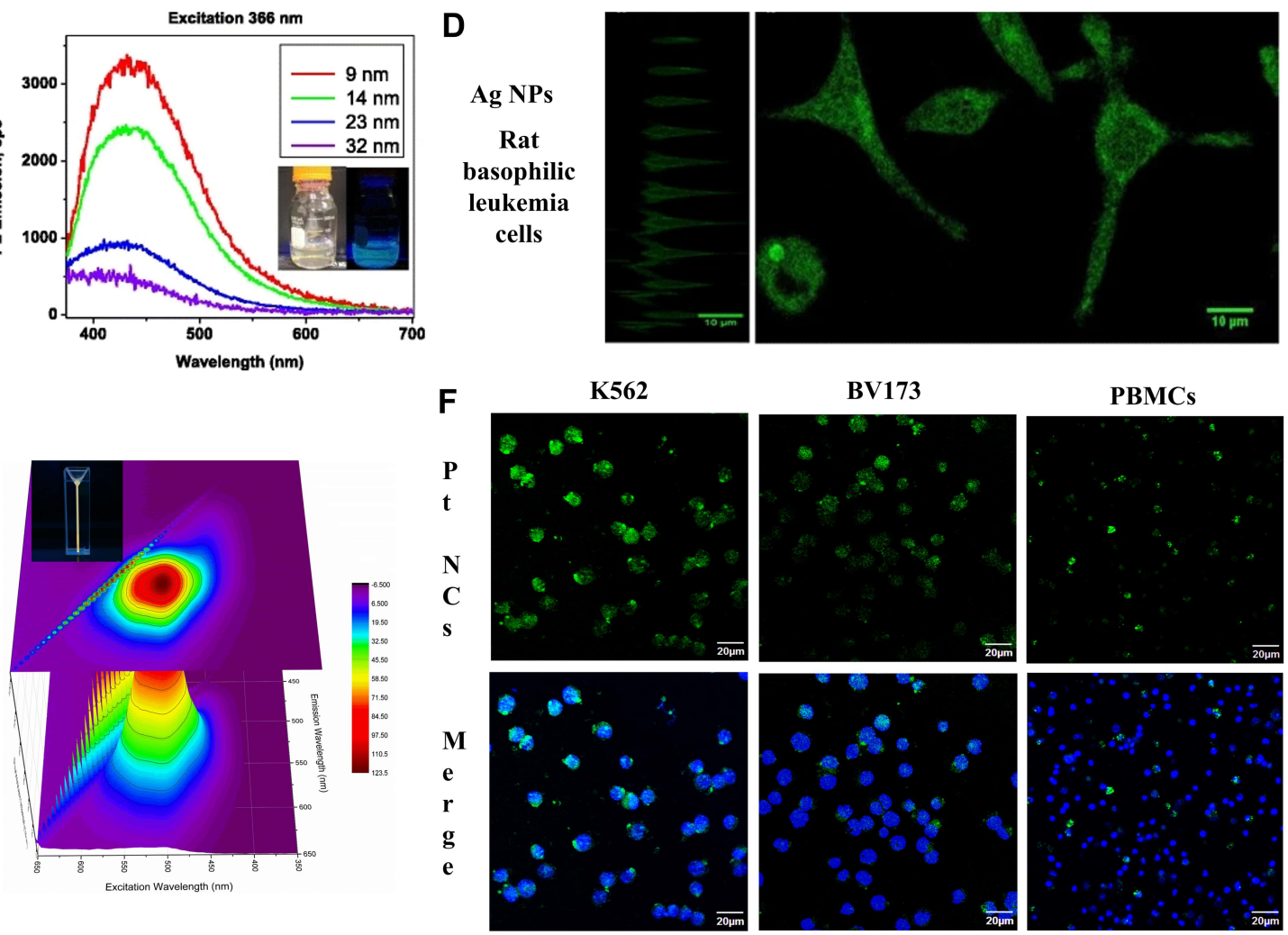

Fig. 3. The fluorescence-based diagnosis of hematological malignancies using metal nanomaterials. (A) $3 \mathrm{D}$ excitation and emission spectrum of multidentate thioether-terminated poly (methacrylic acid) (PTMP-PMAA) stabilized near-infrared (NIR) fluorescent gold nanoclusters (Au NCs) and (B) confocal microscopic images of human umbilical cord blood mononuclear cells (CBMC) and hematopoietic cancer K562 cells cultured with Au NCs for $24 \mathrm{~h}$. Cell nuclei were stained by Hochest 33258. Reprinted major modification with permission from [86], Copyright 2011, American Chemical Society. (C) Photoluminescence emission spectra of Ag NPs with different sizes excited by $366 \mathrm{~nm}$ UV light and (D) confocal microscopic graphs under cross-section (z-stacks) and top view of rat basophilic leukemia cells imaged by synthesized Ag NPs. Reprinted major modification with permission from [87], Copyright 2016, Springer. (E) 3D excitation and emission spectrum of polyethylenimine (PEI)-encased platinum nanoclusters (Pt NCs@PEI) and (F) confocal microscopic images of hematopoietic cancer K562 and BV173 cells as well as peripheral blood mononucleated cells (PBMCs) cultured with Pt NCs@PEI. Cell nuclei were stained by 6-diamidino-2-phenylindole (DAPI). Reprinted major modification with permission from [94], Copyright 2018, Elsevier. 
increased use frequency of CD22 and active agents targeting B cell maturation antigen (BCMA) [109-111]. For example, Au nanourchins (GNUs) with core diameter at 101 $\pm 12 \mathrm{~nm}$ and branch length at $31.9 \pm 12.9 \mathrm{~nm}$, were initially modified by the Raman reporter molecule Nile Blue (NB) and stabilizer polyethylene glycol (PEG) and subsequently conjugated with antiCD19 IgA monoclonal antibodies in order to specifically target CD19 positive leukemic cells [112]. For the acquisition of a nondistinctive cell apoptosis effect, the dosage of antiCD19-PEG-NB-GNU treatment $(0.17 \mathrm{ng} / \mu \mathrm{L})$ was much less than that of antiCD19 alone $(0.56 \mathrm{ng} / \mu \mathrm{L})$. The possible cytotoxic effect was due to high oxidative stress and a blocking in the growth phases of the CCRF-SB cell cycle. Cancer stem cells (CSCs) rarely occur in multiple myeloma because of tumor multiresistance and recurrence [8]. Dou's group studied the influence of antiABCG2 monoclonal antibody (McAb) conjugated with Ag NPs and vincristine (VCR) on myeloma CSCs [113]. They found that $\mathrm{CD} 44+\mathrm{CD} 24-$ cells separated from the murine myeloma cell line SP2/0 had the characteristics of myeloma CSCs and McAb-Ag NPs-VCR showed close to $100 \%$ cell apoptosis, displaying an effective method to eradicate seeds of hematologic malignancies through specifically targeted therapy. FLT3 is another attractive targeting agent, given its overexpression on most leukemia cells as well as the high rate of FLT3 mutations in human leukemia [38]. Astilean group reported the FLT3 inhibitor-Au NPs conjugates were fabricated to targeted delivery of anti-drugs (midostaurin, sorafenib, quizartinib, and lestaurtinib) which have a superior therapeutic effect for $\mathrm{AML}$ comparison with the bare drugs [114].

Generally, nanomedicines have a tendency to extravasate into solid tumor tissue via permeability and retention (EPR) effects, whereas, vascular phase and diffuse localization of hematologic malignancies make the EPR effect less efficient [115]. NM NMs with outstanding chemicophysical and biological properties can be used as the targeted delivery carriers or functional adjuvants in combination with other carriers like liposomes, biocompatible and degradable polymers, magnetic nanocarrier [40]. The establishment of NM NMs-based drug delivery systems (DDS) for the development of targeted therapy has become an innovative breakthrough in the traditional treatment of refractory cancers especially hematopoietic diseases (Fig. 4). Patra et al. [116] proposed a potential AuVel-FA DDS including velcade (Vel) as an anti-cancer drug, folic acid (FA) as a targeting agent and Au NP as a delivery vehicle. Au-Vel-FA was capable of inducing apoptosis against both multiple myeloma U266 and RPMI cells on the premise of maintaining the functional activity of velcade. This form of DDS could be deemed as an equally effective alternative to classical chemotherapeutics and capable of being extended to other malignancies. A triple DDS called DNA-nanosilver-berberine was constructed employing DNA as a carrier Ag NPs as a plasmonic accelera- tor, and berberine as an effective drug for leukemic cancer [117]. DNA-nanosilver-berberine exerted high toxicity against CCRF-CEM cells inducing apoptosis of cells through increased ROS production and executive caspase 3/7 activation. Ag NPs accelerated the electronic transitions of Berberine and adsorbed the high energy emission absorbent in the integration with penetrative light radiation for deeper localized tumors [118].

In a word, either NM NMs-ligand conjugates or NM NMs-based DDS for targeted therapy not only protect drugs against the external environment during the transportation towards the target site, but also increase the blood circulation time and surface multi-functionality. Most important is that the long circulation properties will increase the possibility of medicable drugs encountering malignant cells in peripheral blood which is one of the main target sites in hematologic malignancies [115]. High efficiency, specific selectivity and unique applicability of targeted therapy makes it advantageous for the treatment of hematologic malignancies, however, it is regrettable that targeted therapy has not shown a survival advantage in stage IV disease for many randomized trials, despite its significantly improving patient survival and quality of life [119].

\subsection{Photothermal therapy}

As a form of photodynamic therapy (PDT), photothermal therapy (PTT) uses heat created by electromagnetic radiation to eliminate or ablate tumor cells $[120,121]$. Lightresponsive materials can be activated by light or near infrared (NIR) wavelength radiation, resulting in high affinity for cancer cells through hyperthermia [122]. PTT manifests a prominent role in the treatment of cancer based on its noninvasive, non-contact and low cytotoxic properties. Au NPs are preferred in the photo-based nanomedicine because of specific optical features like SPR $[123,124]$. If Au NPs enter into tumors, they trend to aggregate at the local tumor sites, and absorb energy via a light (wavelength of 700-980 $\mathrm{nm} / 1000-1400 \mathrm{~nm}$ ) irradiation to generate heat which can transfer to tumors without damaging normal cells (Fig. 5A) $[121,125]$. The first attempt for the photothermal therapy employing Au NPs in vitro was proposed by Lapotko et al. [126,127]. They used laser activated nano-thermolysis for cell elimination technology (LANTCET) to target thermolysis of leukemia cells by means of producing microbubbles near the NPs under laser irradiation $[126,127]$. Afterwards, Au nanorod (Au NRs) as one kind of NPs were conjugated with CD33 monoclonal antibodies (Au NRs-CD33) to achieve nano-thermolysis of human acute leukemia cells by a NIR pulsed-laser illumination [128]. The proportion of dead hematopoietic tumor cells went up 3-4 times after the PTT by Au NRs-CD33. Wang's group creatively combined both PDT and PTT approaches by constructing an aptamer switch probe (ASP) connected chlorin e6 (Ce6) photosensitizer onto the surface of Au NRs [129]. ASP was formed as the initial sgc8 leukemia aptamer (OFF state) and polyethy- 


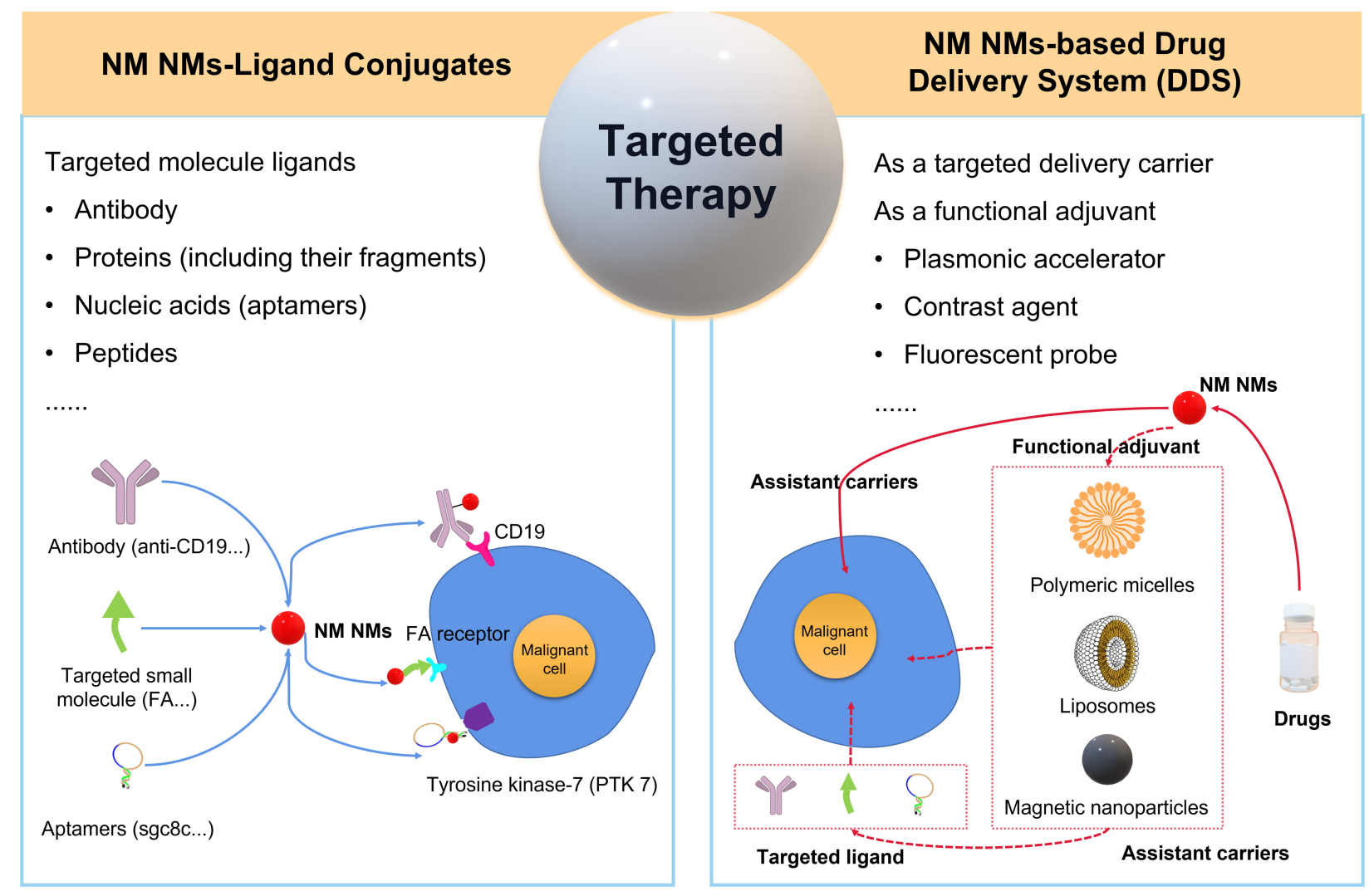

Fig. 4. The schematic targeted therapy of hematologic malignancies using NM NMs to construct the NM NMs-ligand conjugates or NM NMs-based drug delivery system (DDS).

lene glycol (PEG) linked sgc8 (ON state) to turn on/off the fluorescence of the photosensitizer, leading to PDT controlled by singlet oxygen generation (SOG) as stated in Fig. 5B. Au NRs with a length below $100 \mathrm{~nm}$ (Fig. 5C) play a role as a PTT agent by their plasmon resonance absorption (LPRA) in the NIR region. Positive CCRF-CEM (acute lymphoblastic leukemia T-cells) and negative control Ramos cells (acute lymphoblastic leukemia B-cells) were selected to examine the efficiency of PDT/PTT and the result revealed that this multimodal therapy could efficiently kill the CCRF-CEM (cell viability $=39 \%$ ) after $812 \mathrm{~nm} \mathrm{NIR}$ laser irradiation for 10 min compared to nontarget Ramos cells (cell viability $=88 \%$ ) as shown in Fig. 5D,E [129].

Although NM NMs have remarkable success in PTT for various cancers, the limitations such as inapplicability to metastasing tumors and irreversible tumor growth caused by hyperthermia need specific attention in the future.

\section{Conclusion and perspective}

This review summarizes three types of hematological malignancies and current advances in their diagnosis and treatment. Considerable effort has been made on the investigation of NM NMs especially $\mathrm{Au}, \mathrm{Ag}$, Pt NMs as sensitive probes and safe contrast agents to achieve the detection and cellular imaging of hematologic malignancies due to their peculiar physicochemical properties including LSPR, SERS, and size-dependent fluorescence, as well as relatively low cytotoxicity. Moreover, the intrinsic features of NM NMs such as ultrafine size, unique optical and electronical properties, especially the easy modifiable surface have the ability of integrating these with other contrast agents, chemotherapeutic agents, targeted agents and physiotherapy agents to set up innovative chemotherapeutic, targeted and photothermal therapeutic platforms. In spite of the diagnostic and therapeutic effectiveness of NM NMs, several limitations and imperfections of NM NMs remain to be addressed: (1) the accuracy of the NM NMs-based probes need to be further promoted even at trace levels; (2) exploiting the high fluorescent NM NMs-based biomarker in the NIR range in order to eliminate the interference of autofluorescence and improve the biological imaging effect; (3) NM NMs-based nanomedicine is urgently required to solve the problems of short half-lives, lower bioavailability, and resistance to drugs; (4) new theranostic strategies need to be developed not only limited to traditional chemoradiotherapy, but also extended to immunotherapy, targeted therapy and integrated multi-therapy. 
A

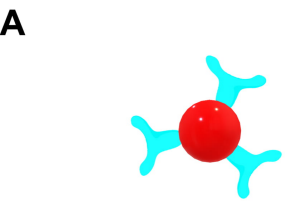

\section{Targeted-ligand} functionated NM NMs

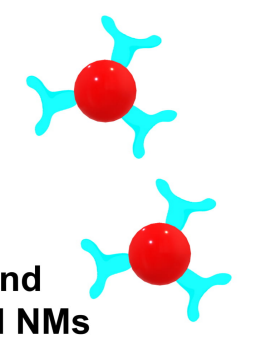

Generate heat To kill the cells

B OFF

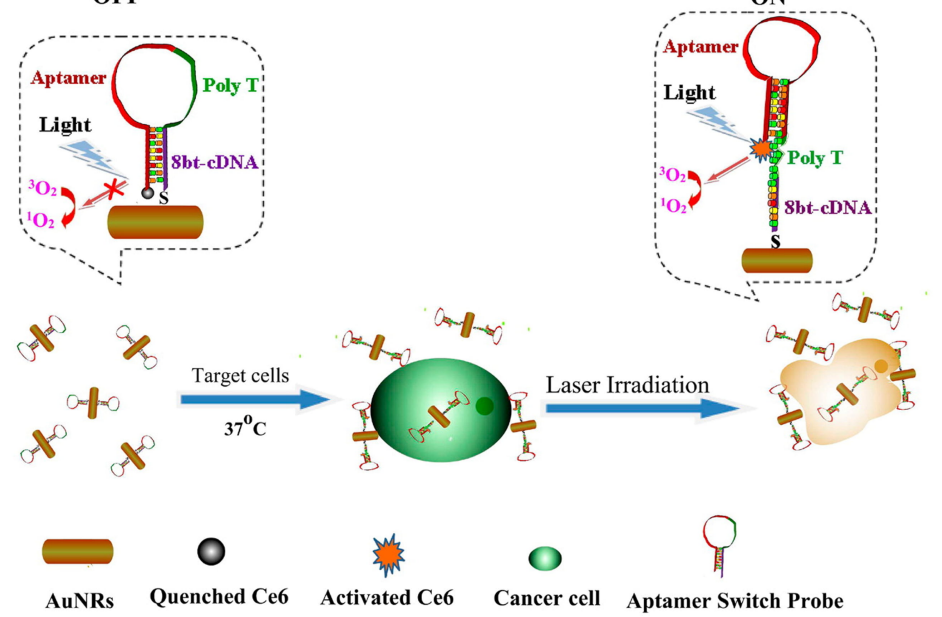

Sequence: 5'-SH-CTA ACC GTT TTT TTT TTT TTT TTT TTT TTT TTT TTT TTT TAT CTA ACT GCT GCG CCG CCG GGA AAA TAC TGT ACG GTT AGA-Ce6-3'

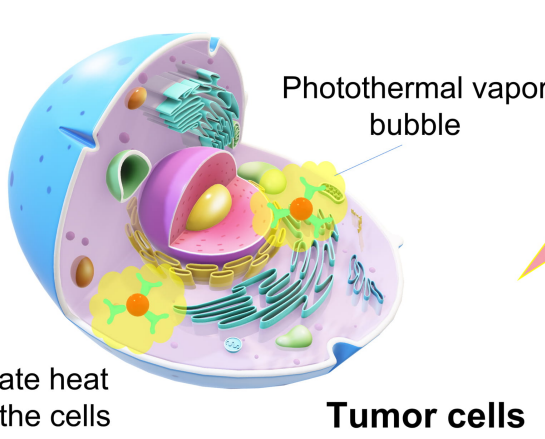

Laser irradiation

C
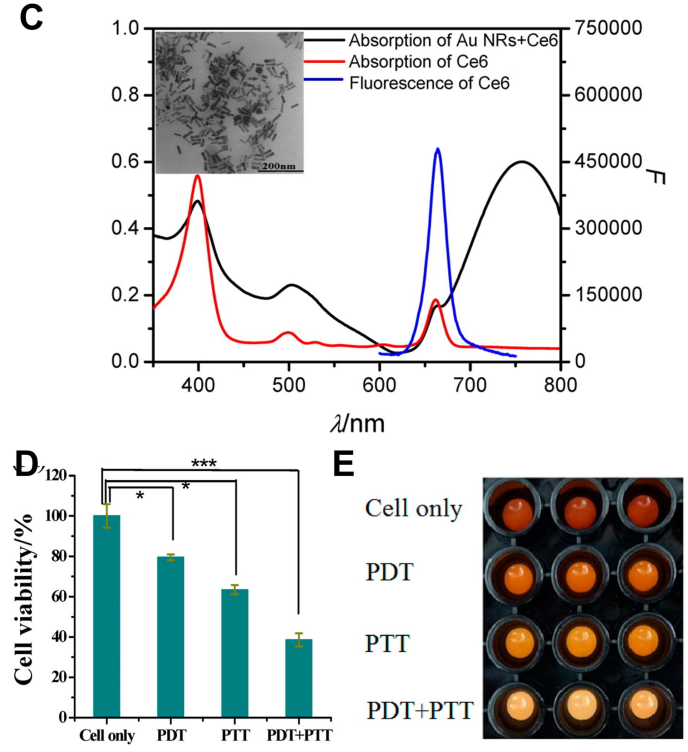

E Cell only PDT PTT PDT+PTT $O-0$

Fig. 5. The photothermal therapy of hematological malignancies using metal nanomaterials. (A) Schematic representation of NM NMs-based photothermal therapy for tumors. (B) Scheme of the construction of an aptamer switch probe (ASP) and photosensitizer Ce6 modified the gold nanorods (Au NRs) to form the ASP-photosensitizer-Au NRs complex for photothermal therapy and photodynamic therapy. (C) Absorption spectra of Au NRs-Ce6 complex and free Ce6, emission spectrum of free Ce6. Inset picture is the transmission electron microscope (TEM) image of Au NRs. (D) Cell viability data and imaging (E) of CCRF-CEM cells incubated with ASP-photosensitizer-Au NRs without light irradiation (cells only), under white light irradiation (PDT), under $812 \mathrm{~nm}$ laser irradiation (PTT), and PDT + PTT, respectively. Cells, $200 \mathrm{k} /$ sample; probes, $0.2 \mathrm{nM} . p$ values were calculated by Student's $t$-test: ${ }^{*} p<0.05, * * p$ $<0.001,{ }^{* * *} p<0.0001, \mathrm{n}=3$. Reprinted major modification with permission from [129], Copyright 2011, American Chemical Society.

\section{Author contribution}

$\mathrm{XH}$ proposed the outline of this review and wrote the manuscript. HMM, XD, ZL, XS collected the related literatures and revised the manuscript. ZX, LZ, TL and HL put forward constructive opinions on this review and participated in the writing of the paper. All authors contributed to the article and approved the submitted version.

\section{Ethics approval and consent to participate}

Not applicable.

\section{Acknowledgments}

Xin Huang gratefully acknowledges the support from "The 2021 Scientific Research and Entrepreneurial StartUps Foundation for the Returned Overseas Chinese Schol- ars, Henan Province", "The 2018 Backbone Teachers of Zhongyuan University of Technology", "Zhengzhou Key Laboratory of Green Dyeing and Finishing Technology", "Henan Collaborative Innovation Centre of Textile and Garment Industry" and "Zhengzhou Key Laboratory of Green Dyeing and Finishing Technology" for their assistance.

\section{Funding}

This work was funded by National Natural Science Foundation of China (Grant No. 21807121), Henan Provincial Science and Technology Research Project (Grant No. 212102310197), Henan Province Foundation for University Key Teacher (Grant No. 2021GGJS107). 


\section{Conflict of interest}

The authors declare no conflict of interest.

\section{References}

[1] Metter DM, Colgan TJ, Leung ST, Timmons CF, Park JY. Trends in the us and Canadian Pathologist Workforces from 2007 to 2017. JAMA Network Open. 2019; 2: e194337.

[2] Bray F, Ferlay J, Soerjomataram I, Siegel RL, Torre LA, Jemal A. Global cancer statistics 2018: GLOBOCAN estimates of incidence and mortality worldwide for 36 cancers in 185 countries. CA - A Cancer Journal for Clinicians. 2018; 68: 394- 424.

[3] Arber DA, Orazi A, Hasserjian R, Thiele J, Borowitz MJ, Le Beau MM, et al. The 2016 revision to the World Health Organization classification of myeloid neoplasms and acute leukemia. Blood. 2016; 127: 2391-2405.

[4] Kansara RR, Speziali C. Immunotherapy in hematologic malignancies. Current Oncology. 2020; 27: S124-S131.

[5] Taylor J, Xiao W, Abdel-Wahab O. Diagnosis and classification of hematologic malignancies on the basis of genetics. Blood. 2017; 130: 410-423.

[6] Juliusson G, Hough R. Leukemia. Progress in Tumor Research. 2016; 43: 87-100.

[7] Mugnaini EN, Ghosh N. Lymphoma. Primary Care. 2016; 43: 661-675.

[8] Kyle RA, Rajkumar SV. Multiple Myeloma. New England Journal of Medicine. 2004; 351: 1860-1873.

[9] Seth R, Singh A. Leukemias in Children. Indian Journal of Pediatrics. 2015; 82: 817-824.

[10] Whitehead TP, Metayer C, Wiemels JL, Singer AW, Miller MD. Childhood Leukemia and Primary Prevention. Current Problems in Pediatric and Adolescent Health Care. 2016; 46: 317-352.

[11] Arber DA, George TI. Why is Hematopathology so Complicated? Surgical Pathology Clinics. 2013; 6: ix.

[12] Krause JR. Who Classification of Tumours of Haematopoietic and Lymphoid Tissues: an Overview. Critical Values. 2009; 2 : 30-32.

[13] Calvo KR, Braylan RC. Advances in diagnostic hematopathology. Seminars in Hematology. 2019; 56: 1.

[14] Hallek M. Chronic lymphocytic leukemia: 2020 update on diagnosis, risk stratification and treatment. American Journal of Hematology. 2019; 94: 1266-1287.

[15] Ansell SM. Hodgkin Lymphoma: Diagnosis and Treatment. Mayo Clinic Proceedings. 2015; 90: 1574-1583.

[16] Klener P, Etrych T, Klener P. Biological Therapy of Hematologic Malignancies: toward a Chemotherapy- free Era. Current Medicinal Chemistry. 2019; 26: 1002-1018.

[17] Atkins S, He F. Chemotherapy and beyond. Infectious Disease Clinics of North America. 2019; 33: 289-309.

[18] Gonella S, Di Giulio P. Delayed Chemotherapy-Induced Nausea and Vomiting in the Hematology Population: A Review of the Literature. Clinical Journal of Oncology Nursing. 2015; 19: 438-443.

[19] Stellitano A, Fedele R, Barilla S, Iaria A, Rao CM, Martino M. Chemotherapy and Cardiotoxicity in Hematologic Malignancies. Current Cancer Drug Targets. 2017; 17: 311-324.

[20] Escrihuela-Vidal F, Laporte J, Albasanz-Puig A, Gudiol C. Update on the management of febrile neutropenia in hematologic patients. Revista Espanola de Quimioterapia. 2019; 32: 55-58.

[21] Singh N, Loren AW. Overview of Hematopoietic Cell Transplantation for the Treatment of Hematologic Malignancies. Clinics in Chest Medicine. 2017; 38: 575-593.

[22] Inamoto Y, Lee SJ. Late effects of blood and marrow transplantation. Haematologica. 2017; 102: 614-625.

[23] Ciurea SO, Cao K, Fernandez-Vina M, Kongtim P, Malki MA, Fuchs E, et al. The European Society for Blood and Marrow
Transplantation (EBMT) Consensus Guidelines for the Detection and Treatment of Donor-specific Anti-HLA Antibodies (DSA) in Haploidentical Hematopoietic Cell Transplantation. Bone Marrow Transplantation. 2018; 53: 521-534.

[24] Im A, Pavletic SZ. Immunotherapy in hematologic malignancies: past, present, and future. Journal of Hematology and Oncology. 2017; 10: 94.

[25] Nelson MH, Paulos CM. Novel immunotherapies for hematologic malignancies. Immunological Reviews. 2015; 263: 90 105.

[26] Cirillo M, Tan P, Sturm M, Cole C. Cellular Immunotherapy for Hematologic Malignancies: Beyond Bone Marrow Transplantation. Biology of Blood and Marrow Transplantation. 2018; 24: 433-442.

[27] Sang W, Zhang Z, Dai Y, Chen X. Recent advances in nanomaterial-based synergistic combination cancer immunotherapy. Chemical Society Reviews. 2019; 48: 37713810 .

[28] Salles G, Barrett M, Foà R, Maurer J, O'Brien S, Valente N, et al. Rituximab in B-Cell Hematologic Malignancies: A Review of 20 Years of Clinical Experience. Advances in Therapy. 2017; 34: 2232-2273.

[29] York IA, Rock KL. Antigen processing and presentation by the class I major histocompatibility complex. Annual Review of Immunology. 1996; 14: 369-396.

[30] Staerz UD, Kanagawa O, Bevan MJ. Hybrid antibodies can target sites for attack by T cells. Nature. 1985; 314: 628-631.

[31] Duell J, Lammers PE, Djuretic I, Chunyk AG, Alekar S, Jacobs I, et al. Bispecific Antibodies in the Treatment of Hematologic Malignancies. Clinical Pharmacology and Therapeutics. 2019; 106: 781-791.

[32] Lucca LE, Dominguez-Villar M. Modulation of regulatory T cell function and stability by co-inhibitory receptors. Nature Reviews Immunology. 2020; 20: 680-693.

[33] Leick MB, Maus MV. Toxicities associated with immunotherapies for hematologic malignancies. Best Practice and Research. Clinical Haematology. 2018; 31: 158-165.

[34] Santomasso B, Bachier C, Westin J, Rezvani K, Shpall EJ. The other Side of CAR T-Cell Therapy: Cytokine Release Syndrome, Neurologic Toxicity, and Financial Burden. American Society of Clinical Oncology educational book / ASCO. American Society of Clinical Oncology. Meeting. 2019; 39: 433-444.

[35] Chung C. Current targeted therapies in lymphomas. American Journal of Health-System Pharmacy. 2019; 76: 1825-1834.

[36] Shimada A. Hematological malignancies and molecular targeting therapy. European Journal of Pharmacology. 2019; 862: 172641.

[37] Safdari Y, Ahmadzadeh V, Farajnia S. CD20-targeting in B-cell malignancies: novel prospects for antibodies and combination therapies. Investigational New Drugs. 2016; 34: 497-512.

[38] Annesley CE, Brown P. The Biology and Targeting of FLT3 in Pediatric Leukemia. Frontiers in Oncology. 2014; 4: 263.

[39] Shanmuganathan N, Hiwase DK, Ross DM. Treatment of chronic myeloid leukemia: assessing risk, monitoring response, and optimizing outcome. Leukemia and Lymphoma. 2017; 58: 2799-2810.

[40] Visani G, Loscocco F, Isidori A. Nanomedicine strategies for hematological malignancies: what is next? Nanomedicine. 2014; 9: 2415-2428.

[41] Egusquiaguirre SP, Igartua M, Hernández RM, Pedraz JL. Nanoparticle delivery systems for cancer therapy: advances in clinical and preclinical research. Clinical and Translational Oncology. 2012; 14: 83-93.

[42] Björnmalm M, Thurecht KJ, Michael M, Scott AM, Caruso F. Bridging Bio-Nano Science and Cancer Nanomedicine. ACS Nano. 2017; 11: 9594-9613. 
[43] Wicki A, Witzigmann D, Balasubramanian V, Huwyler J. Nanomedicine in cancer therapy: challenges, opportunities, and clinical applications. Journal of Controlled Release. 2015; 200: 138-157.

[44] Jain PK, Huang X, El-Sayed IH, El-Sayed MA. Noble metals on the nanoscale: optical and photothermal properties and some applications in imaging, sensing, biology, and medicine. Accounts of Chemical Research. 2008; 41: 1578-1586.

[45] Wilcoxon JP, Abrams BL. Synthesis, structure and properties of metal nanoclusters. Chemical Society Reviews. 2006; 35: 11621194.

[46] Choi HK, Lee M-J, Lee SN, Kim T-H, Oh B-K. Noble metal nanomaterial-Based biosensors for electrochemical and optical detection of viruses causing respiratory illnesses. Frontiers in Chemistry. 2021; 9: 672739 .

[47] Siegel RW. Nanostructured materials -mind over matter-. Nanostructured Materials. 1994; 4: 121-138.

[48] Mori K, Yamashita H. Design of Colloidal and Supported Metal Nanoparticles: their Synthesis, Characterization, and Catalytic Application. Journal of the Japan Petroleum Institute. 2011; 54: $1-14$.

[49] Ghosh SK, Pal T. Interparticle coupling effect on the surface plasmon resonance of gold nanoparticles: from theory to applications. Chemical Reviews. 2007; 107: 4797-4862.

[50] Zhang L, Wang E. Metal nanoclusters: New fluorescent probes for sensors and bioimaging. Nano Today. 2014; 9: 132-157.

[51] Chen L, Wang C, Yuan Z, Chang H. Fluorescent gold nanoclusters: recent advances in sensing and imaging. Analytical Chemistry. 2015; 87: 216-229.

[52] Huang X, Li Z, Yu Z, Deng X, Xin Y. Recent Advances in the Synthesis, Properties, and Biological Applications of Platinum Nanoclusters. Journal of Nanomaterials. 2019; 2019.

[53] Díez I, Ras RHA. Fluorescent silver nanoclusters. Nanoscale. 2011; 3: 1963-1970.

[54] Li J, Zhao T, Chen T, Liu Y, Ong CN, Xie J. Engineering noble metal nanomaterials for environmental applications. Nanoscale. 2015; 7: 7502-7519.

[55] Lu C, Chang F. Polyhedral Oligomeric SilsesquioxaneEncapsulating Amorphous Palladium Nanoclusters as Catalysts for Heck Reactions. ACS Catalysis. 2011; 1: 481-488.

[56] Tang Q, Yang T, Huang Y. Copper nanocluster-based fluorescent probe for hypochlorite. Microchimica Acta. 2015; 182: 23372343.

[57] Medici S, Peana M, Nurchi VM, Lachowicz JI, Crisponi G, Zoroddu MA. Noble metals in medicine: Latest advances Coordination Chemistry Reviews. 2015; 284: 329-350.

[58] Parker JP, Ude Z, Marmion CJ. Exploiting developments in nanotechnology for the preferential delivery of platinum-based anticancer agents to tumours: targeting some of the hallmarks of cancer. Metallomics. 2016; 8: 43-60.

[59] Cassano D, Mapanao A, Summa M, Vlamidis Y, Giannone G, Santi M, et al. Biosafety and Biokinetics of Noble Metals: The Impact of their Chemical Nature. ACS Applied Bio Materials. 2019; 2: 4464-4470.

[60] Yaqoob SB, Adnan R, Khan RMR, Rashid M. Gold, silver, and palladium nanoparticles: A chemical tool for biomedical applications. Frontiers in Chemistry. 2020; 8: 376.

[61] Blanco-Formoso M, Alvarez-Puebla RA. Cancer diagnosis through SERS and other related techniques. International Journal of Molecular Sciences. 2020; 21: 2253.

[62] Schwarzenbach H, Hoon DSB, Pantel K. Cell-free nucleic acids as biomarkers in cancer patients. Nature Reviews Cancer. 2011; 11: 426-437.

[63] He Y, Lin J, Kong D, Huang M, Xu C, Kim T, et al. Current State of Circulating MicroRNAs as Cancer Biomarkers. Clinical Chemistry. 2015; 61: 1138-1155.
[64] Hoshino A, Costa-Silva B, Shen T, Rodrigues G, Hashimoto A, Tesic Mark M, et al. Tumour exosome integrins determine organotropic metastasis. Nature. 2015; 527: 329-335.

[65] Ignatiadis M, Lee M, Jeffrey SS. Circulating Tumor Cells and Circulating Tumor DNA: Challenges and Opportunities on the Path to Clinical Utility. Clinical Cancer Research. 2015; 21: 4786-4800.

[66] Zardavas D, Irrthum A, Swanton C, Piccart M. Clinical management of breast cancer heterogeneity. Nature Reviews Clinical Oncology. 2015; 12: 381-394.

[67] Lertvachirapaiboon C, Baba A, Shinbo K, Kato K. Colorimetric Detection Based on Localized Surface Plasmon Resonance for Determination of Chemicals in Urine. Analytical Sciences. 2021; 37: 929-940.

[68] Amirjani A, Haghshenas DF. Ag nanostructures as the surface plasmon resonance (SPR)-based sensors: a mechanistic study with an emphasis on heavy metallic ions detection. Sensors and Actuators, B: Chemical. 2018; 273: 1768-1779.

[69] Mayer KM, Hafner JH. Localized Surface Plasmon Resonance Sensors. Chemical Reviews. 2011; 111: 3828-3857.

[70] Rycenga M, Cobley CM, Zeng J, Li W, Moran CH, Zhang Q, et al. Controlling the synthesis and assembly of silver nanostructures for plasmonic applications. Chemical Reviews. 2011; 111: 3669-3712.

[71] Valsecchi C, Jones T, Wang C, Lochbihler H, Menezes JW, Brolo AG. Low-Cost Leukemic Serum Marker Screening Using Large Area Nanohole Arrays on Plastic Substrates. ACS Sensors. 2016; 1: 1103-1109.

[72] Benton HP. Cytokines and their receptors. Current Opinion in Cell Biology. 1991; 3: 171-175.

[73] Oh B, Huang N, Chen W, Seo JH, Chen P, Cornell TT, et al. Integrated nanoplasmonic sensing for cellular functional immunoanalysis using human blood. ACS Nano. 2014; 8: 2667-2676.

[74] Kneipp J, Kneipp H, Wittig B, Kneipp K. Novel optical nanosensors for probing and imaging live cells. Nanomedicine. 2010; 6: 214-226.

[75] McNay G, Eustace D, Smith WE, Faulds K, Graham D. Surfaceenhanced Raman scattering (SERS) and surface-enhanced resonance Raman scattering (SERRS): a review of applications. Applied Spectroscopy. 2011; 65: 825-837.

[76] Nolan JP, Sebba DS. Surface-enhanced Raman scattering (SERS) cytometry. Methods in Cell Biology. 2011; 102: 515532.

[77] MacLaughlin CM, Mullaithilaga N, Yang G, Ip SY, Wang C, Walker GC. Surface-enhanced Raman scattering dye-labeled $\mathrm{Au}$ nanoparticles for triplexed detection of leukemia and lymphoma cells and SERS flow cytometry. Langmuir. 2013; 29: 1908 1919.

[78] Alattar N, Daud H, Al-Majmaie R, Zeulla D, Al-Rubeai M, Rice JH. Surface-enhanced Raman scattering for rapid hematopoietic stem cell differentiation analysis. Applied Optics. 2018; 57: E184-E189.

[79] Willets KA. Surface-enhanced Raman scattering (SERS) for probing internal cellular structure and dynamics. Analytical and Bioanalytical Chemistry. 2009; 394: 85-94.

[80] Lentini G, Fazio E, Calabrese F, De Plano LM, Puliafico M, Franco D, et al. Phage-AgNPs complex as SERS probe for U937 cell identification. Biosensors and Bioelectronics. 2015; 74: 398-405.

[81] Nagy-Simon T, Tatar A-S, Craciun A-M, Vulpoi A, Jurj MA, Florea A, et al. Antibody conjugated, Raman tagged hollow gold-silver nanospheres for specific targeting and multimodal Dark-Field/SERS/Two Photon-FLIM Imaging of CD19(+) B Lymphoblasts. ACS Applied Materials \& Interfaces. 2017; 9: 21155-21168.

[82] Lin HH, Chan YC, Chen JW, Chang CC. Aggregation-induced 
emission enhancement characteristics of naphthalimide derivatives and their applications in cell imaging. Journal of Materials Chemistry. 2011; 21: 3170-3177.

[83] Subach FV, Verkhusha VV. Chromophore Transformations in Red Fluorescent Proteins. Chemical Reviews. 2012; 112: 43084327.

[84] Freeman R, Willner I. Optical molecular sensing with semiconductor quantum dots (QDs). Chemical Society Reviews. 2012; 41: 4067-4085

[85] Zhang X, Wang S, Zhu C, Liu M, Ji Y, Feng L, et al. Carbondots derived from nanodiamond: photoluminescence tunable nanoparticles for cell imaging. Journal of Colloid and Interface Science. 2013; 397: 39-44.

[86] Huang X, Luo Y, Li Z, Li B, Zhang H, Li L, et al. Biolabeling Hematopoietic System Cells Using near-infrared Fluorescent Gold Nanoclusters. The Journal of Physical Chemistry C. 2011; 115: 16753-16763.

[87] Kravets V, Almemar Z, Jiang K, Culhane K, Machado R, Hagen $\mathrm{G}$, et al. Imaging of Biological Cells Using Luminescent Silver Nanoparticles. Nanoscale Research Letters. 2016; 11: 30.

[88] Chen A, Holt-Hindle P. Platinum-based nanostructured materials: synthesis, properties, and applications. Chemical Reviews. 2010; 110: 3767-3804.

[89] Huang X, Ishitobi H, Inouye Y. Formation of fluorescent platinum nanoclusters using hyper-branched polyethylenimine and their conjugation to antibodies for bio-imaging. RSC Advances. 2016; 6: 9709-9716.

[90] Xia X, Zhang Y, Wang J. Novel fabrication of highly fluorescent Pt nanoclusters and their applications in hypochlorite assay. RSC Advances. 2014; 4: 25365-25368.

[91] Le Guével X, Trouillet V, Spies C, Jung G, Schneider M. Synthesis of Yellow-Emitting Platinum Nanoclusters by Ligand Etching. The Journal of Physical Chemistry C. 2012; 116: 60476051.

[92] Kawasaki H, Yamamoto H, Fujimori H, Arakawa R, Inada M, Iwasaki Y. Surfactant-free solution synthesis of fluorescent platinum subnanoclusters. Chemical Communications. 2010; 46: 3759-3761.

[93] Tanaka S, Miyazaki J, Tiwari DK, Jin T, Inouye Y. Fluorescent platinum nanoclusters: synthesis, purification, characterization, and application to bioimaging. Angewandte Chemie - International Edition. 2011; 50: 431-435.

[94] Chen X, Zhou J, Yue X, Wang S, Yu B, Luo Y, et al. Selective bio-labeling and induced apoptosis of hematopoietic cancer cells using dual-functional polyethylenimine-caged platinum nanoclusters. Biochemical and Biophysical Research Communications. 2018; 503: 1465-1470.

[95] Zhang Q, Yang M, Zhu Y, Mao C. Metallic Nanoclusters for Cancer Imaging and Therapy. Current Medicinal Chemistry. 2018; 25: 1379-1396.

[96] Hu X, Li F, Noor N, Ling D. Platinum drugs: from Pt (II) compounds, Pt (IV) prodrugs, to Pt nanocrystals/nanoclusters. Science Bulletin. 2017; 62: 589-596.

[97] Rosenberg B, VanCamp L, Trosko JE, Mansour VH. Platinum compounds: a new class of potent antitumour agents. Nature. 1969; 222: 385-386.

[98] Dilruba S, Kalayda GV. Platinum-based drugs: past, present and future. Cancer Chemotherapy and Pharmacology. 2016; 77: $1103-1124$

[99] Barabas K, Milner R, Lurie D, Adin C. Cisplatin: a review of toxicities and therapeutic applications. Veterinary and Comparative Oncology. 2008; 6: 1-18.

[100] Chien C, Yan J, Chiu W, Wu T, Liu C, Lin S. Caged Pt nanoclusters exhibiting corrodibility to exert tumor-inside activation for anticancer chemotherapeutics. Advanced Materials. 2013; 25: 5067-5073.
[101] Xia H, Li F, Hu X, Park W, Wang S, Jang Y, et al. PHSensitive Pt Nanocluster Assembly Overcomes Cisplatin Resistance and Heterogeneous Stemness of Hepatocellular Carcinoma. ACS Central Science. 2016; 2: 802-811.

[102] Guo D, Zhu L, Huang Z, Zhou H, Ge Y, Ma W, et al. Antileukemia activity of PVP-coated silver nanoparticles via generation of reactive oxygen species and release of silver ions. Biomaterials. 2013; 34: 7884-7894.

[103] Guo D, Zhao Y, Zhang Y, Wang Q, Huang Z, Ding Q, et al. The Cellular Uptake and Cytotoxic Effect of Silver Nanoparticles on Chronic Myeloid Leukemia Cells. Journal of Biomedical Nanotechnology. 2014; 10: 669-678.

[104] Bhattacharya R, Patra CR, Verma R, Kumar S, Greipp PR, Mukherjee P. Gold Nanoparticles Inhibit the Proliferation of Multiple Myeloma Cells. Advanced Materials. 2007; 19: 711716.

[105] Kumari P, Kumari N, Jha AK, Singh KP, Prasad K. Nyctanthes arbortristis mediated synthesis of silver nanoparticles: Cytotoxicity assay against THP-1 human leukemia cell lines. AIP Conference Proceedings. AIP Publishing LLC. 2018; 1953: 030071.

[106] Shafek R, Michael H, Sayed A, Ibrahim A, Al-sayed A. Phytochemical study, antioxidant and cytotoxic activities of Brassica rapa L. leaves extract and its silver nanoparticles. Egyptian Journal of Chemistry. 2018; 61: 237-247.

[107] Amgoth C, Singh A, Santhosh R, Yumnam S, Mangla P, Karthik R, et al. Solvent assisted size effect on AuNPs and significant inhibition on K562 cells. RSC Advances. 2019; 9: 33931-33940.

[108] Di Pietro P, Strano G, Zuccarello L, Satriano C. Gold and Silver Nanoparticles for Applications in Theranostics. Current Topics in Medicinal Chemistry. 2016; 16: 3069-3102.

[109] Sharkey RM, Behr TM, Mattes MJ, Stein R, Griffiths GL, Shih $\mathrm{LB}$, et al. Advantage of residualizing radiolabels for an internalizing antibody against the B-cell lymphoma antigen, CD22. Cancer Immunology, Immunotherapy. 1997; 44: 179-188.

[110] Yu B, Jiang T, Liu D. BCMA-targeted immunotherapy for multiple myeloma. Journal of Hematology and Oncology. 2020; 13: 125.

[111] Yu JX, Upadhaya S, Tatake R, Barkalow F, Hubbard-Lucey VM. Cancer cell therapies: the clinical trial landscape. Nature Reviews Drug Discovery. 2020; 19: 583-584.

[112] Tatar A, Jurj A, Tomuleasa C, Florea A, Berindan-Neagoe I, Cialla-May D, et al. CD19-targeted, Raman tagged gold nanourchins as theranostic agents against acute lymphoblastic leukemia. Colloids and Surfaces B: Biointerfaces. 2019; 184: 110478.

[113] Dou J, He X, Liu Y, Huang Z, Yang C, Shi F, et al. Targeted therapeutic effect of anti-ABCG2 antibody combined with nano silver and vincristine on mouse myeloma cancer stem cells. Journal of Nanoparticle Research. 2013; 15: 2127.

[114] Simon T, Tomuleasa C, Bojan A, Berindan-Neagoe I, Boca S, Astilean S. Design of FLT3 Inhibitor - Gold Nanoparticle Conjugates as Potential Therapeutic Agents for the Treatment of Acute Myeloid Leukemia. Nanoscale Research Letters. 2015; 10: 466.

[115] Torchilin V. Multifunctional and stimuli-sensitive pharmaceutical nanocarriers. European Journal of Pharmaceutics and Biopharmaceutics. 2009; 71: 431-444.

[116] Patra CR, Verma R, Kumar S, Greipp PR, Mukhopadhyay D, Mukherjee P. Fabrication of gold nanoparticle for potential application in multiple myeloma. Journal of Biomedical Nanotechnology. 2008; 4: 499-507.

[117] Grebinyk A, Yashchuk V, Bashmakova N, Gryn D, Hagemann T, Naumenko A, et al. A new triple system DNA-NanosilverBerberine for cancer therapy. Applied Nanoscience. 2019; 9: 945-956.

[118] Zheng Y, Hunting DJ, Ayotte P, Sanche L. Radiosensitization 
of DNA by gold nanoparticles irradiated with high-energy electrons. Radiation Research. 2008; 169: 19-27.

[119] Socinski MA. Targeted therapy vs chemotherapy: which has had more impact on survival in lung cancer? The significant impact of chemotherapy in lung cancer. Clinical Advances in Hematology and Oncology. 2014; 12: 763-768.

[120] Wang Y, Meng H-M, Li Z. Near-infrared inorganic nanomaterial-based nanosystems for photothermal therapy. Nanoscale. 2021; 13: 8751-8772.

[121] Yang W, Lyu Q, Zhao J, Cao L, Hao Y, Zhang H. Recent advance in near-infrared/ultrasound-sensitive 2D-nanomaterials for cancer therapeutics. Science China Materials. 2020; 63: 2397-2428

[122] Kim K, Khang D. Past, present, and future of anticancer nanomedicine. International Journal of Nanomedicine. 2020; 15: 5719-5743.

[123] Menon JU, Jadeja P, Tambe P, Vu K, Yuan B, Nguyen KT. Nanomaterials for photo-based diagnostic and therapeutic applications. Theranostics. 2013; 3: 152-166.

[124] Austin LA, Mackey MA, Dreaden EC, El-Sayed MA. The optical, photothermal, and facile surface chemical properties of gold and silver nanoparticles in biodiagnostics, therapy, and drug de- livery. Archives of Toxicology. 2014; 88: 1391-1417.

[125] Jaque D, Martínez Maestro L, del Rosal B, Haro-Gonzalez P, Benayas A, Plaza JL, et al. Nanoparticles for photothermal therapies. Nanoscale. 2014; 6: 9494-9530.

[126] Lapotko D, Lukianova E, Potapnev M, Aleinikova O, Oraevsky A. Elimination of leukemic cells from human transplants by laser nano-thermolysis. Photons Plus Ultrasound: Imaging and Sensing 2006: The Seventh Conference on Biomedical Thermoacoustics, Optoacoustics, and Acousto-optics. International Society for Optics and Photonics. 2006; 6086: 60860I.

[127] Lapotko D. Plasmonic nanoparticle-generated photothermal bubbles and their biomedical applications. Nanomedicine. 2009; 4: 813-845.

[128] Liopo A, Conjusteau A, Konopleva M, Andreeff M, Oraevsky A. Photothermal therapy of acute leukemia cells in the nearinfrared region using gold nanorods CD-33 conjugates. Optical Interactions with Tissue and Cells XXII. International Society for Optics and Photonics. 2011; 7897: 789710.

[129] Wang J, Zhu G, You M, Song E, Shukoor MI, Zhang K, et al. Assembly of aptamer switch probes and photosensitizer on gold nanorods for targeted photothermal and photodynamic cancer therapy. ACS Nano. 2012; 6: 5070-5077. 